\title{
Glauber's coherent states and the semiclassical propagator
}

H. G. Solari

Citation: Journal of Mathematical Physics 27, 1351 (1986); doi: 10.1063/1.527142

View online: https://doi.org/10.1063/1.527142

View Table of Contents: http://aip.scitation.org/toc/jmp/27/5

Published by the American Institute of Physics

\section{Articles you may be interested in}

Semiclassical representations of electronic structure and dynamics

The Journal of Chemical Physics 120, 579 (2004); 10.1063/1.1630963

Semiclassical treatment of spin system by means of coherent states Journal of Mathematical Physics 28, 1097 (1987); 10.1063/1.527554

Multiconfigurational quantum propagation with trajectory-guided generalized coherent states

The Journal of Chemical Physics 144, 094106 (2016); 10.1063/1.4942926

Frozen Gaussians: A very simple semiclassical approximation

The Journal of Chemical Physics 75, 2923 (1981); 10.1063/1.442382

Ab initio multiple cloning algorithm for quantum nonadiabatic molecular dynamics

The Journal of Chemical Physics 141, 054110 (2014); 10.1063/1.4891530

Initial-value semiclassical propagators for the Wigner phase space representation: Formulation based on the interpretation of the Moyal equation as a Schrödinger equation

The Journal of Chemical Physics 143, 244110 (2015); 10.1063/1.4938235

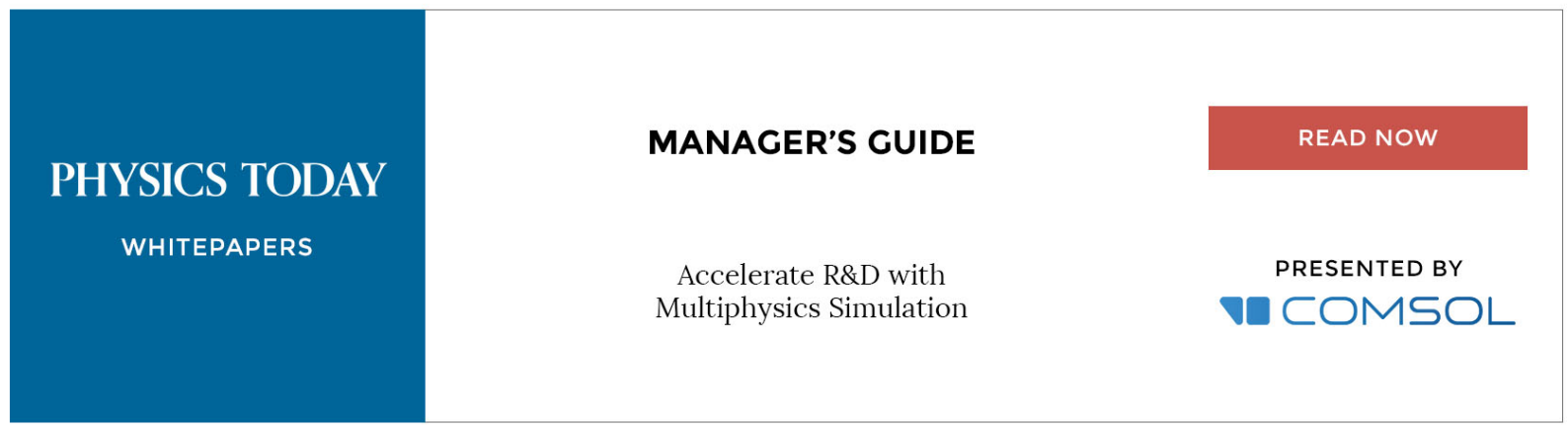




\title{
Glauber's coherent states and the semiclassical propagator
}

\author{
H. G. Solaria) \\ Departamento de Física, Facultad de Ciencias Exactas y Naturales, Universidad de Buenos Aires, 1428 \\ Buenos Aires, Argentina
}

(Received 28 March 1985; accepted for publication 3 January 1986)

\begin{abstract}
This paper studies the classical propagator on Glauber's coherent states in a generalized $P$-form. A full class of equivalent expressions labeled by a complex number is found through a direct WKB expansion. The study of matrix elements of the propagator between coherent states shows that they match Klauder's classical expression but are in some disagreement in the semiclassical order with expression derived through path integral procedures. Arguments are given that favor the present results. The properties of the time propagator with respect to symmetry operations are stated. Possible extensions are briefly discussed.
\end{abstract}

\section{INTRODUCTION}

Semiclassical-like approaches to quantum problems have received renewed attention in the last years because of their ability to obtain useful descriptions of large systems like heavy ions ${ }^{1}$ and also due to the efforts towards understanding the role of chaos in quantum dynamics. ${ }^{2}$ Furthermore semiclassical approaches have their proper place in mathematical physics.

The harmonic oscillator coherent states, or Glauber coherent states (GCS's), ${ }^{3}$ are well known as the most classical quantum states, i.e., they minimize the Heisenberg uncertainty relationship. Because of this fact, it is expected that they may make semiclassical approaches easier. From another point of view, they are related to the coset representatives of the special nilpotent group ${ }^{4,5}$ and form an overcomplete basis, which makes room for a Bargmann ${ }^{6}$ space. This property provides a way for generalizations and a great amount of structure.

The earlier works on the semiclassical propagator (SP) ${ }^{7,8}$ were not very successful in finding a direct relation between the SP and the classical trajectories in phase space, because of the necessity of complexifying the position and momentum in order to fulfill the boundary conditions, or alternatively because of the necessary introduction of an auxiliary field. ${ }^{9}$ In contrast with these earlier works, which concern the matrix elements of the SP between GCS (or more general coherent states), Suzuki ${ }^{10}$ proposes, on an heuristic basis, an approach to the classical kernel of the propagator in the $P$-form. We recall that $Q$ - and $P$-forms relating operators with coherent states were proposed from the beginning by Glauber ${ }^{3}$ (see also Sec. II and Ref. 5). Suzuki's approach is fully developed with respect to the classical trajectories in phase space.

In this work we study Suzuki's propagator through a direct WKB method. ${ }^{11}$ This procedure allows us to obtain a generalized form of the classical expression and also go further towards the semiclassical one, which does not coincide with that conjectured by Suzuki ${ }^{10}$ (Sec. II). The equivalence of these expressions with Klauder's ${ }^{7}$ is shown in Sec. III, where we also compare our semiclassical formula with

\footnotetext{
a) Fellow of the Consejo Nacional de Investigaciones Científicas y Técnicas (CONICET) of Argentina.
}

the equivalent one by Kuratsuji and Mizobuchi ${ }^{12,13}$ for spin systems. Section IV is devoted to showing the invariance property of the SP related to the Hamiltonian symmetries (this property was observed in some special situations by Levit ${ }^{9}$ and Levit et al. ${ }^{14}$ ). In Sec. V we develop some examples and finally Sec. VI is reserved for the conclusions and perspectives.

\section{THE SEMICLASSICAL PROPAGATOR}

\section{A. P-form of the classical propagator}

The matrix elements of an operator with respect to a GCS is a function called the $Q$-form of the operator $\hat{O}$,

$$
\mathscr{O}_{Q}\left(\alpha, \beta^{*}\right) \equiv\langle\beta|\hat{O}| \alpha\rangle \text {, }
$$

where $|\alpha\rangle$ is a GCS,

$$
\begin{aligned}
& \left.|\alpha\rangle \equiv \exp \left(-\alpha \alpha^{*} / 2\right) \mid \alpha\right), \\
& \mid \alpha)=\sum_{n}(n !)^{-1 / 2} \alpha^{n}|n\rangle .
\end{aligned}
$$

We distinguish here between unnormalized states (normal parentheses) and normalized ones (brackets), the states $|n\rangle$ are the Fock basis states of the harmonic oscillator and $\alpha$ is a complex number, $\alpha=(q+i p) / \sqrt{2}$. The GCS $|\alpha\rangle$ also have the following properties:

$$
|\alpha\rangle=\exp \left(\alpha a^{\dagger}-\alpha^{*} a\right)|0\rangle=\exp \left(-\alpha \alpha^{*} / 2+\alpha a^{\dagger}\right)|0\rangle,
$$

$a^{\dagger}|\alpha\rangle=\exp \left(-\alpha \alpha^{*} / 2\right) \frac{\partial \mid \alpha)}{\partial \alpha}$,

$a|\alpha\rangle=\alpha|\alpha\rangle$,

$\exp \left(-i \epsilon a^{\dagger} a\right)|\alpha\rangle=|\alpha \exp (-i \epsilon)\rangle$,

where $a^{\dagger}(a)$ is the creation (destruction) operator and $|0\rangle$ the ground state of the harmonic oscillator, the expression (2.4c) shows that the GCS's are eigenvectors of the destruction operator.

In addition to the $Q$-form some operators may be written in a diagonal from, ${ }^{3}$ for example, density operators representing statistical mixtures of pure coherent states. This representation is called the $P$-form and deals with operators like

$$
\hat{o}=\int\left[d \alpha \wedge d \alpha^{*} \mathscr{O}_{P}\left(\alpha, \alpha^{*}\right) \mid \alpha\right)(\alpha \mid] .
$$


We note that this $P$-form does not include Suzuki's propagators and seems to be too restrictive for our proposals. We are going to consider here operators $\hat{B}$ of the form

$$
\hat{B}=\int\left[d g \wedge d \alpha^{*} B\left(g, \alpha^{*}\right) \mid g\right)(\alpha \mid],
$$

where $g=g\left(\alpha, \alpha^{*}\right)$.

It is necessary to demand, for a faithful generalization, that the matrix elements of $\hat{B}$ between coherent states be finite figures, which simply means

$$
\left|\int\left[d g \wedge d \alpha^{*} B\left(g, \alpha^{*}\right) \exp \left(g \phi^{*}+\alpha^{*} \xi\right)\right]\right|<\infty,
$$

for arbitrary but fixed $\phi$ and $\xi$.

It is important to note that several different (in appearance) expressions may represent the same operator. This situation will happen at least each time that the integration path $(-\infty<\operatorname{Re} \alpha, \operatorname{Im} \alpha \leqslant \infty)$ may be deformed in a complex way. We have established enough conditions in the Appendix in order that the equivalence holds. The essential requirements are that $B\left(g, \alpha^{*}\right)$ be a holomorphic function of $g$ and $\alpha^{*}$, and $g\left(\alpha, \alpha^{*}\right)$ be extensible in a holomorphic way to $g\left(z, \alpha^{*}\right)$. If this hypothesis holds, the operator $B(2.5)$ may be written in the form

$\hat{B}=\int\left[\left.d z \wedge d \alpha^{*} \frac{\partial g}{\partial z}\right|_{\alpha^{*}} B\left(g\left(z, \alpha^{*}\right), \alpha^{*}\right) \mid g\right)(\alpha \mid]$,

where $i\left(z-\alpha^{*}\right)$ and $\left(z+\alpha^{*}\right)$ go from $-\infty+i 0$ to $\infty+i 0$.

As useful examples we write the identities

$\left.\hat{I}=\frac{1}{\pi} \int_{-\infty}^{\infty} d(\operatorname{Re} \alpha) \int_{-\infty}^{\infty} d(\operatorname{Im} \alpha) e^{-z \alpha^{*}} \mid z\right)(\alpha \mid$,

where $z=\delta+\alpha$ for any complex number $\delta$ (this formula has been proved in the Appendix), and the evolution operator for the Hamiltonian $H=\epsilon a^{\dagger} a$, which reads [applying (2.4d) and (2.7)]

$$
\begin{aligned}
\exp (-i \hat{H} t) & =\exp (-i \hat{H} t) \hat{I} \\
& \left.=\frac{1}{\pi} \iint d(\operatorname{Re} \alpha) d(\operatorname{Im} \alpha) e^{-z \alpha^{*}} \mid z_{t}\right)(\alpha \mid,
\end{aligned}
$$

with $z_{t}=e^{-i \epsilon t} z$.

This last formula suggests the starting point of our approach for the evolution operator $\hat{U}(t), t=t_{f}-t_{i}$, for a time-independent Hamiltonian. We are going to look for a semiclassical approach to $\hat{U}(t)$ assuming that the propagator may be written in the form

$$
\left.\hat{U}(t)=\int d \alpha \wedge d \alpha^{*} \mathscr{U}\left(\gamma_{t}, \alpha^{*}, t\right) \mid \gamma_{t}\right)(\alpha \mid,
$$

where $\gamma_{t}$ is related with $\alpha$ and $\alpha^{*}$ by a time-dependent mapping.

The semiclassical approach centers attention on the states with large mean values of position and momentum. We then introduce an order parameter $N$, which will help us to identify terms in the asymptotic series and is supposed to be large, in the form

$$
\alpha=\sqrt{N} \beta .
$$

The parameter $N$ plays the role of $1 / \hbar$ and later will be taken as unity.

The Hamiltonian is assumed to be of the form

$$
\hat{H}=N \sum_{n m} b_{n m} N^{-(n+m) / 2}\left(a^{\dagger}\right)^{n} a^{m}
$$

and, taking into account Eq. (2.4b), it acts over a GCS as

$$
\left.\hat{H} \mid \alpha)=N \sum_{n m} b_{n m} N^{-(n+m) / 2} \alpha^{m} \frac{\partial^{n}}{\partial \alpha^{n}} \mid \alpha\right) .
$$

The starting point for establishing $\mathscr{U}\left(\gamma_{t}, \alpha^{*}, t\right)$ is the Schrödinger evolution equation

$$
\hat{U}=-i \hat{H} \hat{U} \text {. }
$$

Taking into account the expression (2.8) for $\hat{U}(t)$ and the action of $\hat{H}$ on a coherent state (2.11), we obtain

$$
\begin{aligned}
0= & \left(\frac{\partial}{\partial t}+i \hat{H}\right) \hat{U}(t) \\
= & \int\left[\frac{d \mathscr{U}}{d t} \mid \gamma_{t}\right)\left(\alpha\left|+\mathscr{U}\left(\frac{\partial}{\partial t}+i H\right)\right| \gamma_{t}\right)(\alpha \mid] d \alpha \wedge d \alpha^{*}, \\
0= & \int\left(\frac{\partial \mathscr{U}}{\partial t}+\frac{\partial \mathscr{U}}{\partial \gamma_{t}} \dot{\gamma}_{t}+\mathscr{U} \dot{\gamma}_{t} \frac{\partial}{\partial \gamma_{t}}\right. \\
& \left.+i \mathscr{U} N \sum_{n m} b_{n m} N-(n+m) / 2 \gamma_{t}^{m} \frac{\partial^{n}}{\partial \gamma_{t}^{n}}\right) \\
& \left.\times \mid \gamma_{t}\right)\left(\alpha \mid d \alpha \wedge d \alpha^{*},\right.
\end{aligned}
$$

where $\dot{\gamma}_{t}=d \gamma_{t} / d t$. Introducing the Jacobian

$$
J \equiv\left(\frac{\partial \alpha}{\partial \gamma_{t}}\right)_{\alpha^{*}, t},
$$

integrating by parts (assuming that the surface at $|\alpha|=\infty$ do not contribute), and considering

$$
\mathscr{U}\left(\gamma_{t}, \alpha^{*}, t\right)=\exp \left(i N S\left(\gamma_{t}, \alpha^{*}, t\right)\right) \tilde{\mathscr{U}},
$$

where $\tilde{\mathscr{U}}$ is expanded in the form

$$
\tilde{\mathscr{U}}=\sum_{n=1} N^{-n} \tilde{\mathscr{U}}_{n+1},
$$

we are able to write the following expression:

$$
\begin{aligned}
& N \int\left(i J N \mathscr{U} \frac{\partial S}{\partial t}+J e^{i N S} \frac{\partial \tilde{\mathscr{U}}}{\partial t}-\mathscr{U} \frac{\partial}{\partial z_{t}}\left(\dot{z}_{t} J\right)\right. \\
& \quad+i N \sum_{n m}\left[b_{n m}(-N)^{-n}\right. \\
& \left.\left.\quad \times \sum_{k=0}^{n}\left(\begin{array}{l}
n \\
k
\end{array}\right)\left(\frac{\partial^{k} e^{i N S}}{\partial z_{t}^{k}}\right) \frac{\partial^{n-k}}{\partial z_{t}^{n-k}}\left(\tilde{\mathscr{U}} J z_{t}^{m}\right)\right]\right) \\
& \left.\quad \times \mid \sqrt{N} z_{t}\right)\left(\sqrt{N} \beta \mid d z_{t} \wedge d \beta^{*},\right.
\end{aligned}
$$

where we have performed a change of coordinates in the form of Eq. (2.9) and adopted the notation that partial derivatives are considered to be taken with respect to the variables $\left(z_{t}, \beta^{*}, t\right)$ except when another situation is explicitly indicated.

Identifying the coefficients of $N^{2}$ we get the classical equations

$$
-\frac{\partial S}{\partial t}+\mathscr{H}\left(z_{t},-i \frac{\partial S}{\partial z_{t}}, t\right)=0,
$$

where 


$$
\mathscr{H}\left(z, \beta^{*}\right)=\sum_{n m} b_{n m} z^{n} \beta^{* m}=\frac{(\beta|\hat{H}| z)}{(\beta \mid z)} .
$$

Equations (2.17) are highly undetermined as soon as $z_{t}(\beta$, $\left.\beta^{*}, t\right)$ is an undetermined function. This situation reflects in part the overcompleteness of the coherent states.

A solution for (2.17) may be found requiring that $S$ be an action in a Hamiltonian set of equations, i.e., we require that

$$
\begin{aligned}
& -i \frac{\partial S}{\partial z_{t}}=y_{t}^{*}, \\
& i \ddot{z}_{t}=\left.\frac{\partial \mathscr{H}}{\partial y_{t}^{*}}\left(z_{t}, y_{t}^{*}\right)\right|_{z_{t}} .
\end{aligned}
$$

Demanding that $\hat{H} \hat{U}=\hat{U} \hat{H}$, we obtain, in an equivalent form, Eq. (2.18), the last formulas of the Hamiltonian system

$$
\begin{aligned}
& -i \frac{\partial S}{\partial \beta^{*}}=w_{t_{i}}, \\
& i \frac{d \beta^{*}}{d t_{i}}=\left.\frac{\partial \mathscr{H}}{\partial w_{t_{i}}}\left(w_{t_{i}}, \beta^{*}\right)\right|_{\beta^{*}},
\end{aligned}
$$

where $t_{i}$ is the starting time of the evolution.

As soon as the Hamiltonian has been considered to be time independent, it is natural to require $\hat{U}\left(t_{f}, t_{i}\right)$ to be only a function of $t=\left(t_{f}-t_{i}\right)$, and then it follows that

$$
\mathscr{H}\left(z_{t}, y^{*}\right)=\mathscr{H}\left(w_{t_{i}}, \beta^{*}\right) \text {. }
$$

The set of equations [ (2.17) and (2.18)] allows us to integrate $S$, which turns out to be

$S=\int_{t_{i}}^{t_{f}}\left(i y^{*} \dot{z}_{t}-\mathscr{H}\left(z_{t}, y^{*}\right)\right) d t+\beta^{*} z_{z_{i}}+$ const ,

where $z_{t} \equiv w_{t}$ and $y_{t}^{*} \equiv \beta_{i}^{*}$ fulfill the Hamiltonian equation (2.18b).

The action $S$ reflects some undetermination related to the initial conditions for $z_{t}$ and $y^{*}$. This problem may be studied comparing the classical propagator at $t=0$ with the identity (2.7), which implies that

$$
z_{t_{i}}=\beta+\delta,
$$

with $\delta$ an arbitrary complex number. The full set of equations are summarized in Table $\mathrm{I}$.

The classical propagator reads

$$
\left.\hat{U}_{\mathrm{cl}}=\int \exp \left(i S\left(z_{t}, \beta^{*}, t\right)\right) / i \pi \mid z_{t}\right)\left(\beta \mid d \beta \wedge d \beta^{*}\right.
$$

and the particular choice of $\delta=0$ in (2.20) brings us to the Suzuki propagator. ${ }^{10}$

\section{B. The semiclassical propagator}

We are now going to analyze the first correction to the classical approach, which comes from the coefficient of $N^{1}$ in (2.16). The correction $\tilde{\mathscr{U}}_{2}$ must satisfy the following equation:

$$
\begin{aligned}
0= & -\tilde{\mathscr{U}}_{2} J \frac{\partial \dot{z}_{t}}{\partial z_{t}}+J \frac{d \tilde{\mathscr{U}}_{2}}{d t}-i \tilde{\mathscr{U}}_{2} J \frac{\partial^{2} \mathscr{H}\left(z_{t}, \beta_{t}^{*}\right)}{\left(\partial z_{t} \partial \beta_{t}^{*}\right)} \\
& -0.5 \tilde{\mathscr{U}}_{2} J \frac{\partial^{2} \mathscr{H}\left(z_{t}, \beta_{t}^{*}\right)}{\left(\partial \beta_{t}^{*}\right)^{2}} \frac{\partial^{2} S}{\left(\partial z_{t}\right)^{2}}
\end{aligned}
$$

Considering $\tilde{\mathscr{U}}_{2}=e^{i S_{2}}$ with the initial condition $S_{2}\left(z_{0}, \beta^{*}\right.$, $0)=0$, the second-order action must satisfy the equation

$$
\begin{aligned}
\frac{i d S_{2}}{d t}= & \left(i \frac{\partial^{2} \mathscr{H}\left(z_{t}, \beta_{t}^{*}\right)}{\partial z_{t} \partial \beta_{t}^{*}}\right. \\
& \left.+0.5 \frac{\partial^{2} \mathscr{H}\left(z_{t} \beta_{t}^{*}\right)}{\partial \beta_{t}^{* 2}} \frac{\partial^{2} S}{\partial z_{t}^{2}}+\frac{\partial \dot{z}_{t}}{\partial z_{t}}\right),
\end{aligned}
$$

which, after some handling, is transformed to

$$
i \frac{d S_{2}}{d t}=-0.5 \frac{d}{d t}\left[\ln \left(\frac{\partial^{2} S}{\left(\partial \beta^{*} \partial z_{t}\right)}\right)\right]+0.5 i \frac{\partial^{2} \mathscr{H}\left(z_{t}, \beta_{t}^{*}\right)}{\partial z_{t}+\partial \beta_{t}^{*}} \text {. }
$$

Time integration of (2.23) and (2.24), taking into account the initial condition, yields the first correction to the classical kernel of the time propagator as

$$
\tilde{\mathscr{U}}_{2}= \pm\left(i \frac{\partial^{2} S}{\partial z_{t} \partial \beta^{*}}\right)^{1 / 2} \exp \left[0.5 i \int_{t_{i}}^{t_{f}} \frac{\partial^{2} \mathscr{H}\left(z_{t} \beta_{t}^{*}\right)}{\partial z_{t} \partial \beta_{t}^{*}} d t\right]
$$

It is interesting to note here that while the first term may be identified with the square root of the Jacobian $J$, Eq. (2.14),

$$
-i \frac{\partial^{2} S}{\partial z_{t} \partial \beta^{*}}=\frac{\partial \beta_{t}^{*}}{\partial \beta^{*}}=\frac{\partial z_{0}}{\partial z_{\imath}}=J
$$

an additional exponential term appears. The semiclassical propagator then reads

$$
\begin{aligned}
\hat{U}_{\mathrm{sc}}= & \int \frac{d \beta \wedge d \beta^{*}}{i \pi} J-1 / 2 \exp \left(0.5 i \int_{t_{i}}^{t_{f}} d t \frac{\partial^{2} \mathscr{H}\left(z_{t}, \beta_{t}^{*}\right)}{\partial z_{t} \partial \beta_{t}^{*}}\right) \\
& \left.\times \exp \left(i S\left(z_{t}, \beta^{*}, t\right)\right) \mid z_{t}\right)(\beta \mid .
\end{aligned}
$$

In this formula we also have the freedom to choose $\delta=0$ in (2.20) identifying $z_{t}=\beta_{t}=\left(\beta_{t}^{*}\right)^{*}$.

The expression for $\tilde{\mathscr{U}}_{2}(2.25)$ shows that it is in special situations, as the one considered in Sec. V, that the correction depends only on time. This last assertion was Suzuki's conjecture ${ }^{10}$ (however suppossed to be valid in every case). That is, Suzuki wrote his semiclassical propagator in the form

TABLE I. Full set of equations of the Hamiltonian classical system.

$$
\begin{aligned}
& \frac{\partial S}{\partial t_{f}}=-\frac{\partial S}{\partial t_{i}}=\mathscr{H}\left(z_{t}, \beta_{t_{f}}^{*}\right) \\
& -i \frac{\partial S}{\partial z_{t_{f}}}=y^{*} \equiv \beta_{t_{f}^{*}}^{*} \\
& -i \frac{\partial S}{\partial \beta^{*}}=z_{t_{t}} \equiv z_{0} \\
& \ddot{z}_{t}=\frac{\partial \mathscr{H}\left(z_{t}, \beta_{t}^{*}\right)}{\partial \beta_{t}^{*}} \\
& i \dot{\beta}_{t}^{*}=\frac{-\partial \mathscr{H}\left(z_{t}, \beta_{t}^{*}\right)}{\partial z_{t}} \\
& z_{0}=\beta+\delta \\
& S=\int_{t_{t}}^{t_{f}}\left(i \beta_{t}^{*} \dot{z}_{t}-\mathscr{H}\left(z_{t}, \beta_{i}^{*}\right)\right) d t+\beta^{*} z_{0}+\text { const }
\end{aligned}
$$


$\left.\hat{U}=n(t) \int d \beta \wedge d \beta^{*} \exp \left(i S\left(\beta_{t}, \beta_{t}^{*}, t\right)\right) \mid \beta_{t}\right)(\beta \mid$,

where the secondary kernel $\tilde{\mathscr{U}}_{2}\left(z_{t}, \beta^{*}, t\right)$ does not depend on the trajectory and is partially represented by the timedependent $c$-number $n(t)$.

\section{MATRIX ELEMENTS OF THE SEMICLASSICAL PROPAGATOR}

The matrix elements of the semiclassical propagator with respect to GCS have been studied by Klauder ${ }^{7}$ and later by Levit ${ }^{9}$ and Blaizot and Orland. ${ }^{15}$ It is useful to make contact with this approach from the semiclassical propagator of Eq. (2.27); this proposal only requires the evaluation of the integral by a method consistent with the semiclassical approximation like the saddle point method. ${ }^{16}$

Taking into account the order parameter $N$ again, we have to evaluate

$$
\left(\phi\left|\hat{U}_{\mathrm{sc}}\right| \xi\right)=\int \frac{d \beta \wedge d \beta^{*}}{i \pi} \exp \left\{N\left(i S+z_{t} \phi^{*}+\xi B^{*}\right)\right\} .
$$

The saddle point is characterized by the set of equations

$$
\begin{aligned}
& \phi^{*}=\beta_{t}^{*}, \\
& \xi=z_{0} .
\end{aligned}
$$

These equations cannot be fulfilled for an arbitrary $\delta$, Eq. (2.20), because the equations of motion (2.18) only admit one complex initial condition; the satisfaction of (3.2) would require complexifying the position and momentum as in Refs. 7 and 15. We rather fix the value of $\delta$ in order to make possible the solution of (3.2), noting that the same problem and also the same solutions appear when one evaluates the matrix elements of the identity, (2.7b), by the saddle point method in the form

$$
(\phi|I| \xi)=\exp \left(\phi^{*} g+\xi \alpha^{*}-g \alpha^{*}\right),
$$

where

$$
\alpha^{*}=\phi^{*}, \quad g=\xi,
$$

which fix the value of $\delta$ in the form $\delta=\xi-\phi$, producing the semiclassical evaluation of the matrix elements of the identity by

$$
(\phi \mid \xi)=\exp \left(\phi^{*} \xi\right) .
$$

The classical evaluation of (3.1) reads

$$
\left(\phi\left|\hat{U}_{\mathrm{sc}}\right| \xi\right)=\exp \left(i S+\xi \beta^{*}+\phi^{*} z_{t}\right) \equiv \exp (i F),
$$

where we have defined the function

$F\left(\xi, \phi^{*}\right)=\int_{t_{i}}^{t_{f}} d t\left(i \beta_{t}^{*} \dot{z}_{t}-\mathscr{H}\left(z_{t}, \beta *_{t}^{*}\right)\right)-i z_{t_{f}} \phi^{*}$,

whose partial derivatives are

$$
\begin{aligned}
& i \frac{\partial F\left(\xi, \phi^{*}, t\right)}{\partial \xi}=z_{t_{f}}, \\
& i \frac{\partial F\left(\xi, \phi^{*}, t\right)}{\partial \phi^{*}}=\beta_{t_{i}}^{*} .
\end{aligned}
$$

The equations of motion that arise from the variation

$$
\delta(F)=0
$$

are

$$
\begin{aligned}
& i \ddot{z}_{t}=\frac{\partial \mathscr{H}\left(z_{t}, \beta_{t}^{*}\right)}{\partial \beta_{t}^{*}}, \quad z_{t_{i}}=\xi, \\
& -i \beta_{t}^{*}=\frac{\partial \mathscr{H}\left(z_{t}, \beta_{t}^{*}\right)}{\partial z_{t}}, \quad \beta_{t}^{*}=\phi^{*} .
\end{aligned}
$$

Equations ( 3.3 ) and (3.4) were also found by Klauder ${ }^{7}$ with boundary conditions in both ends $\left(t_{i}\right.$ and $\left.t_{f}\right)$ and are in contrast with Eqs. (2.18) and (2.19) because of the difference in these conditions.

Expanding the exponent in Eq. (3.1) around the saddle point up to the second order and evaluating $\tilde{\mathscr{U}}_{2}$ at this point, we obtain the next-order approximation. Following this procedure and after some handling we get

$$
\begin{aligned}
\left(\phi\left|\hat{U}_{\mathrm{sc}}\right| \xi\right) \simeq & \exp \left(i F+0.5 i \int_{t_{i}}^{t_{f}} d t \frac{\partial^{2} \mathscr{H}\left(z_{t}, \beta_{t}^{*}\right)}{\partial z_{t} \partial \beta_{t}^{*}}\right) \\
& \times\left(-i \frac{\partial^{2} F}{\partial z_{t} \partial \beta^{*}}\right)^{1 / 2},
\end{aligned}
$$

which must be considered together with (3.3) and (3.4).

We may compare this last expression with the one obtained in Refs. 12 and 13 for spin systems by means of a path integral approach. We note at a first glance that the present expression is a little more closed because it is not expressed in terms of the eigenvalues of a Sturm-Liouville problem. ${ }^{12,13}$ Despite this small difference we note that for $t_{f}=t_{i}$ we obtain

$$
\left(\phi\left|\hat{\mathscr{U}}_{\mathrm{sc}}\right| \xi\right) \simeq \exp \left(-\phi^{*} \xi\right)=(\phi|\hat{I}| \xi)=(\phi \mid \xi),
$$

which is the correct figure, while in a simple example presented in Ref. 13 (considering the $J_{z}$ Hamiltonian) the matrix elements for the semiclassical propagator evaluated at $t_{f}=t_{i}$ diverges. It has been suggested (Ref. 17, p. 248) that this behavior may be due to a too restricted election of the paths.

This procedure completes the evaluation of the semiclassical matrix elements, which also presents an extra phase coming from the second-order term. The formulas (2.27) and (3.5) are easily found as the exact figures for the harmonic oscillator, nontrivial examples are shown in Sec. V.

\section{PERIODIC HAMILTONIANS}

The expression (2.27) of the SP is very well suited for studying the way in which symmetries are dealt with in the semiclassical approach. Symmetries are important by themselves and also may be reflected in the requantization formulas obtained from the coherent states-SP as found by Levit $e t$ $a l$. in the frame of the Lipkin model. ${ }^{14}$ Approximate formulas for the energies of a system may be obtained by the Gutzwiller quantization method ${ }^{18-21}$ that assigns a relevant role to the closed orbits and their neighborhoods. ${ }^{18} \mathrm{We}$ are not developing this point in the present work, but let us mention that the approach to the SP here developed may allow a better understanding of chaotic motion and its relationship with quantal energies just because this approach is based on actual classical trajectories in phase space (and not only on stationary paths).

Turning to the symmetry problem we are going to sup- 
pose that the Hamiltonian $\hat{H}$ does commute with the operator $\hat{G}$,

$$
\hat{G}=\exp \left(\alpha a^{\dagger}-\alpha^{*} a\right),
$$

for some fixed $\alpha$, not necessarily infinitesimal,

$$
[\hat{G}, \hat{H}]=0 \text {. }
$$

The action of the operator $G$ on the SP, $\hat{U}_{\mathrm{sc}}$, reads

$$
\left.\hat{G} \hat{U}_{\mathrm{sc}}=\int \frac{d \beta \wedge d \beta^{*}}{i \pi} \tilde{U}_{2} e^{i s} \hat{G} \mid z_{t}\right)(\beta \mid,
$$

while the action of $\hat{G}$ on an (unnormalized) GCS is

$$
\left.\hat{G} \mid z)=\exp \left(-\alpha \alpha^{*} / 2-z \alpha^{*}\right) \mid z+\alpha\right) \text {. }
$$

From this equation and (4.2) we get

$$
\begin{aligned}
\mathscr{H}\left(z_{t}, \beta_{t}^{*}\right) & =\frac{\left(\beta_{t}|\hat{H}| z_{t}\right)}{\left(\beta_{t} \mid z_{t}\right)}=\frac{\left(\beta_{t}+\alpha|\hat{H}| z_{t}+\alpha\right)}{\left(\beta_{t}+\alpha \mid z_{t}+\alpha\right)} \\
& =\mathscr{H}\left(z_{t}+\alpha \beta_{t}^{*}+\alpha^{*}\right) \equiv \mathscr{H}\left(u_{t}, v_{t}\right) .
\end{aligned}
$$

It follows that the pair of functions

$$
\begin{aligned}
& u_{t} \equiv z_{t}+\alpha, \\
& v_{t}^{*} \equiv \beta_{t}^{*}+\alpha,
\end{aligned}
$$

is also a solution of Eqs. (2.18b) and (2.18d) with starting points at

$$
u_{t_{i}}=\beta+\alpha+\delta
$$

and

$$
v^{*}=\beta^{*}+\alpha^{*} .
$$

From the periodicity of $\mathscr{H},(4.5)$, the periodicity of the phase portrait with respect to the shifting by $\alpha$ also follows. It is easy to verify by straightforward integration from the expression of the action $S$ in (2.20) that its simultaneous evaluation at the points $\left(z_{t}, \beta^{*}\right)$ and $\left(u_{t}, v^{*}\right)$ gives the relation

$i S\left(z_{t}, \beta^{*}, t\right)=i S\left(u_{t}, v^{*}, t\right)-\alpha \alpha^{*}+\alpha v^{*}+\alpha^{*} u_{t}$,

which in turn allows us to verify the following relationship between the second derivatives of $S$ :

$$
\frac{\partial^{2} S\left(u_{t}, v^{*}, t\right)}{\partial u_{t} \partial v^{*}}=\frac{\partial^{2} S\left(z_{t}, \beta^{*}, t\right)}{\partial z_{t} \partial \beta^{*}}
$$

Equations (4.8) and (4.9) allow us to express $\hat{G} \hat{U}_{\mathrm{sc}}$ in (4.3) through a change to the new variables $v=\beta+\alpha$ and $v^{*}=(\beta+\alpha)^{*}$, as

$$
\begin{aligned}
\hat{G} \hat{U}_{\mathrm{sc}}= & \int \frac{d v \wedge d v^{*}}{i \pi} \tilde{\mathscr{U}}_{2}\left(u_{t}, v^{*}, t\right) \exp \left(i S\left(u_{t}, v^{*}, t\right)\right) \\
& \left.\times \mid u_{t}\right)\left(v \mid \hat{G}=\hat{U}_{\mathrm{sc}} \hat{G},\right.
\end{aligned}
$$

which proves that both the classical and the semiclassical propagators do commute with the shifting operator $\hat{G}$, Eq. (4.1), if the Hamiltonian $\hat{H}$ does. Let us remark that the proof that we develop here is based on group theoretical arguments and may be directly extended to Suzuki's propagators.

\section{AN EXAMPLE: THE QUADRATIC HAMILTONIAN}

We are going to consider the SP for a system with a Hamiltonian of the form

$$
\hat{H}=\epsilon a^{\dagger} a+0.5\left(v\left(a^{\dagger}\right)^{2}+v^{*}(a)^{2}\right) .
$$

Associating with $\hat{H}$ the classical Hamiltonian $\mathscr{H}$,

$$
\mathscr{H}\left(z \beta^{*}\right)=\epsilon z \beta^{*}+0.5\left(v z^{2}+v^{*} \beta^{* 2}\right),
$$

and the equations of motion

$$
\begin{aligned}
& i \dot{z}_{t}=\epsilon z_{t}+v \beta_{t}^{*}=\left.\frac{\partial \mathscr{H}}{\partial \beta_{t}^{*}}\right|_{z_{t}}, \quad z_{0}=\beta_{0}+\delta, \\
& -i \beta_{t}^{*}=\epsilon \beta_{t}^{*}+v^{*} z_{t}=\left.\frac{\partial \mathscr{H}}{\partial z_{t}}\right|_{\beta_{t}^{*}}, \quad \beta_{0}^{*}=\beta^{*} .
\end{aligned}
$$

The action (2.19) for this system simply reads

$$
S=0.5 i\left(z_{0} \beta^{*}+z_{t} \beta_{t}^{*}\right),
$$

where

$$
z_{t}=z_{0} \cos (\omega t)-i / \omega\left(\epsilon z_{0}+v \beta^{*}\right) \sin (\omega t),
$$

and

$$
\begin{aligned}
& \beta_{i}^{*}=\beta^{*} \cos (\omega t)+i / \omega\left(\epsilon \beta^{*}+v^{*} z\right) \sin (\omega t), \\
& \omega=\left(\epsilon^{2}-v^{*} v\right)^{1 / 2}
\end{aligned}
$$

are the solutions of (5.3). The action in (5.5a) may be explicitly written in terms of $\left(z_{t}, \beta^{*}, t\right)$ in the form

$$
S=\frac{i\left(\beta^{*} z_{t}+0.5 i / \omega\left(\nu \beta^{* 2}+v^{*} z_{t}^{2}\right) \sin (\omega t)\right)}{\cos (\omega t)-i \epsilon / \omega-\sin (\omega t)}
$$

and its second derivative with respect to $z_{t}$ and $\beta^{*}$ is

$$
\frac{\partial^{2} S}{\partial z_{t} \partial \beta^{*}}=\frac{1}{\cos (\omega t)-i \epsilon / \omega \sin (\omega t)} / 1=i J
$$

which in this case does not depend on the variables $z_{t}$ and $\beta^{*}$.

The semiclassical propagator in the $P$-form (2.27) reads in this particular model

$$
\begin{aligned}
\hat{U}_{\mathrm{sc}}= & \int d \beta \wedge d \beta^{*} \\
& \times \exp \left(-\left(\beta^{*} z_{t}+0.5 i / \omega\left(v^{*} z_{t}^{2}+v \beta^{* 2}\right) \sin (\omega t)\right) / J\right) \\
& \left.\times J^{-1 / 2} /(i \pi) \exp (0.5 i \epsilon t) \mid z_{t}\right)(\beta \mid .
\end{aligned}
$$

It may be directly checked ${ }^{10}$ that for the quadratic Hamiltonian (5.1) the SP matches the exact one as usual. ${ }^{17}$

\section{CONCLUSIONS}

We have studied the classical propagator expressed in terms of the Glauber coherent states in the $\boldsymbol{P}$-form. It has been shown in this context that one of them matches Suzuki's classical expressions. In this case, the kernel of the propagator is fully determined by the classical trajectories in phase space (labeled by their initial conditions). We have also developed explicitly the formulas to the next order, which contribute not only to the square root of the Jacobian but also to an additional time-dependent phase.

We have shown that the expression developed here is equivalent (to the same order in the asymptotic series) to the Klauder formulas for the matrix elements of the semiclassical propagator. Going to these matrix elements we have to match two complex boundary conditions, which forces us to fix the complex parameter $\delta$ that labels the elements of the class of the semiclassical propagators. This requirement as 
well as the existence of a complete class of SP ( $P$-form) is fully related to the overcompleteness of the coherent states.

We have also shown that the SP preserves the dynamic symmetries of the Hamiltonian.

Generalization to systems of several particles (or to higher dimensions) seems to be immediate, and because the most important points are based on the overcompleteness of the coherent states and their group structure we believe that many expressions may be extended to more general coherent states, in particular the symmetry properties of the Suzuki's propagator are always valid. In addition to this common structure, we observe that the proofs developed in Sec. II make use of several special properties of GCS's, not shared by other sets of coherent states; because of this situation an actual generalization beyond GCS requires some work, which is now in progress.

\section{ACKNOWLEDGMENT}

I am grateful to Dr. E. Susana Hernández for helpful discussions and a careful reading of the manuscript.

\section{APPENDIX: EQUIVALENCE RELATIONS FOR P.FORM OPERATORS}

We wish to establish enough conditions for the equivalence of any two different generalized $P$-forms of an operator. In other words, we are going to sketch a proof for the following theorem.

Theorem: Let $\mathscr{O}(q, p)$ be a function such that

$$
\int \mathscr{O}(q, p) \exp (A(q, p)) d q d p=\gamma, \quad|\gamma|<\infty,
$$

where the integration is extended to the whole plane $(q, p)$ and $A$ is a linear function of $(q, p)$ with complex range. In addition, let $\mathcal{O}(q, p)$ be extensible to a holomorphic function of $z_{1}$ and $z_{2}, \mathscr{O}\left(z_{1}, z_{2}\right)$, in a domain $D$ which includes the plane $(q+i 0 ; p+i 0)$. Then

$$
\int d z_{1} \int d z_{2} \mathscr{O}\left(z_{1}, z_{2}\right) \exp \left(A\left(z_{1}, z_{2}\right)\right)=\gamma,
$$

where $\Gamma_{1}$ and $\Gamma_{2}$ are curves that belong to $D$ and extend from $-\infty+i 0$ to $\infty+i 0$.

Proof: As $\mathscr{O}\left(z_{1}, z_{2}\right)$ is a holomorphic function in $D$,

$$
\begin{aligned}
\Delta\left(z_{2}\right) & \equiv \int_{-\infty}^{+\infty} \mathscr{O}\left(q, z_{2}\right) \exp \left(A\left(q, z_{2}\right)\right) d q \\
& =\int_{r_{1}} \mathscr{O}\left(z_{1}, z_{2}\right) \exp \left(A\left(z_{1}, z_{2}\right)\right) d z_{1},
\end{aligned}
$$

where the equality holds by virtue of Cauchy's theorem on holomorphic functions. The function $\Delta\left(z_{2}\right)$ will be also holomorphic because of the hypothesis on $O\left(z_{1}, z_{2}\right)$ and assuming that the derivation under the integration sign is allowed. Applying Cauchy's theorem again, we obtain

$$
\gamma=\int_{\Gamma_{2}} d z_{2} \int d z_{1} \mathscr{O}\left(z_{1}, z_{2}\right) \exp \left(A\left(z_{1}, z_{2}\right)\right),
$$

which completes the proof.

The possibility of generalizing the complex paths for each integral depends upon the convergence conditions of
$\mathscr{O}\left(z_{1}, z_{2}\right)$ for large $\left|\operatorname{Re} z_{1}\right|$ and $\left|\operatorname{Re} z_{2}\right|$. It is reasonable to expect that

$$
\lim _{A \rightarrow \infty} \int_{\Gamma^{\prime}(A)} \mathscr{O}\left(z_{1}, z_{2}\right) \exp \left(A\left(z_{1}, z_{2}\right)\right) d z_{1}=0,
$$

if $\Gamma^{\prime}(A)$ is a curve which goes from $A$ to $A+i f(A)$ and $\left|\lim _{A \rightarrow \infty} f(A)\right|$ is finite, because $\mathscr{O}(q, p)$ goes to zero when $q$ goes to infinity faster than any exponential.

We are going to show two corollaries of the theorem.

Corollary 1: $\mathscr{O}^{\prime}\left(\alpha, \alpha^{*}\right)=\mathscr{O}(q, \quad p)$, where $\alpha=(q+i p) / \sqrt{2}$, and we restrict ourselves to paths such that $z_{1}-i z_{2}=\alpha * / \sqrt{2}$, we obtain the identity

$$
\begin{aligned}
& \int \mathscr{O}^{\prime}\left(\alpha, \alpha^{*}\right) \exp \left(A^{\prime}\left(\alpha, \alpha^{*}\right)\right) d q d p \\
& =\int \mathscr{O}^{\prime}\left(\alpha, \alpha^{*}\right) \exp \left(A^{\prime}\left(\alpha, \alpha^{*}\right)\right) \frac{d \alpha \wedge d \alpha^{*}}{i} \\
& =\int_{\Gamma_{1}} \int_{\Gamma_{2}} \mathscr{O}^{\prime}\left(g, \alpha^{*}\right) \exp \left(A^{\prime}\left(g, \alpha^{*}\right)\right) \frac{d g \wedge d \alpha^{*}}{i},
\end{aligned}
$$

where

$$
A^{\prime}\left(\alpha, \alpha^{*}\right)=A(q, p) \text { and } g=\left(z_{1}+i z_{2}\right) / \sqrt{2} \text {. }
$$

Corollary 2: The identity may be written in terms of coherent states in the form

$$
\begin{aligned}
\hat{I} & \left.=\frac{1}{\pi} \int_{-\infty+i a}^{\infty+i a} d p \int_{-\infty+i b}^{\infty+i b} d q \exp \left(-z \alpha^{*}\right) \mid z\right)(\alpha \mid \\
& \left.=\frac{1}{\pi} \int_{-\infty}^{\infty} d p \int_{-\infty}^{\infty} d q \exp \left(-z \alpha^{*}\right) \mid z\right)(\alpha \mid
\end{aligned}
$$

where $z=\alpha+(a+i b)$.

The proof follows by applying the theorem and Corollary 1 to the matrix elements of $I$ between coherent states and noting for the second equation that

$$
\begin{aligned}
0 & \left.=\int_{-\infty}^{-\infty+i a} d p \exp \left(-z \alpha^{*}\right) \mid z\right)(\alpha \mid \\
& \left.=\int_{\infty}^{\infty+i a} d p \exp \left(-z \alpha^{*}\right) \mid z\right)(\alpha \mid
\end{aligned}
$$

and

$$
\begin{aligned}
0 & \left.=\int_{-\infty}^{-\infty+i b} d q \exp \left(-z \alpha^{*}\right) \mid z\right)(\alpha \mid \\
& \left.=\int_{\infty}^{\infty+i b} d q \exp \left(-z \alpha^{*}\right) \mid z\right)(\alpha \mid
\end{aligned}
$$

because $\exp \left(-z \alpha^{*}+A\left(z, \alpha^{*}\right)\right)$ ( $A$ linear) goes to zero as $|\alpha|$ goes to infinity as a quadratic exponential.

We note that this kind of deformation is necessary when we evaluate matrix elements by means of the saddle point method $^{16}$ and has been formally applied in Refs. 7, 12, 13, and 15 calling the procedure the complexification of the variables $q$ and $p$.

'J. W. Negele, Rev. Mod. Phys. 54, 913 (1982).

${ }^{2}$ Y. Weissman and J. Jortner, J. Chem. Phys. 77, 1486 (1982).

${ }^{3}$ R. J. Glauber, Phys. Rev. 131, 2766 (1963).

${ }^{4}$ A. Perelomov, Commun. Math. Phys. 26, 222 (1972).

${ }^{5}$ R. Gilmore, Rev. Mex. Fis. 23, 143 (1974). 
${ }^{6}$ V. Bargmann, Commun. Pure Appl. Math. 14, 187 (1961).

7J. Klauder, Phys. Rev. D 19, 2349 (1979).

8J. Klauder, Ann. Phys. NY 11, 123 (1960).

'S. Levit, Phys. Rev. C 21, 1594 (1980).

${ }^{10}$ T. Suzuki, Nucl. Phys. A 398, 557 (1983).

${ }^{11}$ A. Messiah, Mechanica Cuantica (Tecnos, Madrid, 1965).

${ }^{12}$ H. Kuratzuji and Y. Mizobuchi, Phys. Lett. A 82, 279 (1981).

${ }^{13}$ H. Kuratzuji and Y. Mizobuchi, J. Math. Phys. 22, 757 (1981).

${ }^{14}$ S. Levit, J. W. Negele, and L. Paltiel, Phys. Rev. C 21, 1603 (1980).
${ }^{15}$ J. P. Blaizot and H. Orland, Phys. Rev. C 24, 1740 (1981).

${ }^{16}$ E. T. Copson, Asymptotic Expansions (Cambridge U. P., Cambridge, $1965)$.

${ }^{17}$ L. S. Schulman, Techniques and Applications of Path Integration (Wiley, New York, 1981).

${ }^{18}$ M. C. Gutzwiller, J. Math. Phys. 12, 343 (1971).

${ }^{19}$ M. C. Gutzwiller, J. Math. Phys. 8, 1979 (1967).

${ }^{20}$ M. C. Gutzwiller, J. Math. Phys. 10, 1004 (1969).

${ }^{21}$ M. C. Gutzwiller, J. Math. Phys. 11, 1791 (1970). 